\title{
AKARI North Ecliptic Pole Deep Survey
}

\section{Revision of the catalogue via a new image analysis ${ }^{\star}$}

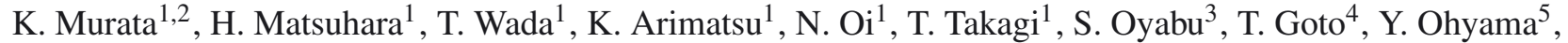

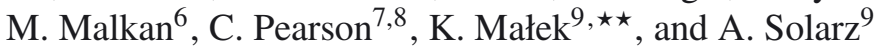 \\ ${ }^{1}$ Institute of Space and Astronautical Science, Japan Aerospace Exploration Agency, Sagamihara, 229-8510 Kanagawa, Japan \\ e-mail: murata@ir.isas.jaxa.jp \\ 2 Department of Space and Astronautical Science, The Graduate University for Advanced Studies, Japan \\ 3 Graduate School of Science, Nagoya University, Furo-cho, Chikusa-ku, Nagoya, 464-8602 Aichi, Japan \\ ${ }^{4}$ Dark Cosmology Centre, Niels Bohr Institute, University of Copenhagen, 2100 Copenhagen, Denmark \\ 5 Institute of Astronomy and Astrophysics, Academia Sinica, PO Box 23-141, 10617 Taipei, Taiwan, R.O.C. \\ ${ }^{6}$ Department of Physics and Astronomy, UCLA, Los Angeles, CA 90095-1547, USA \\ 7 Astrophysics Group, Department of Physics, The Open University, Milton Keynes, MK7 6AA, UK \\ 8 RAL Space, Rutherford Appleton Laboratory, Chilton, Didcot, Oxfordshire OX11 0QX, UK \\ 9 Department of Physics and Astrophysics, Nagoya University, Furo-cho, Chikusa-ku, 464-8602 Nagoya, Japan \\ Received 19 March 2013 / Accepted 27 August 2013
}

\section{ABSTRACT}

\begin{abstract}
Context. We present the revised near- to mid-infrared catalogue of the AKARI North Ecliptic Pole deep survey. The survey has the unique advantage of continuous filter coverage from 2 to $24 \mu \mathrm{m}$ over nine photometric bands, but the initial version of the survey catalogue leaves room for improvement in the image analysis stage; the original images are strongly contaminated by the behaviour of the detector and the optical system.

Aims. The purpose of this study is to devise new image analysis methods and to improve the detection limit and reliability of the source extraction.

Methods. We removed the scattered light and stray light from the Earth limb, and corrected for artificial patterns in the images by creating appropriate templates. We also removed any artificial sources due to bright sources by using their properties or masked them out visually. In addition, for the mid-infrared source extraction, we created detection images by stacking all six bands. This reduced the sky noise and enabled us to detect fainter sources more reliably. For the near-infrared source catalogue, we considered only objects with counterparts from ground-based catalogues to avoid fake sources. For our ground-based catalogues, we used catalogues based on the CFHT/MegaCam $z^{\prime}$ band, CFHT/WIRCam $K$ s band and Subaru/Scam $z^{\prime}$ band. Objects with multiple counterparts were all listed in the catalogue with a merged flag for the AKARI flux.

Results. The detection limits of all mid-infrared bands were improved by $\sim 20 \%$, and the total number of detected objects was increased by $\sim 2000$ compared with the previous version of the catalogue; it now has 9560 objects. The $5 \sigma$ detection limits in our catalogue are $11,9,10,30,34,57,87,93$, and $256 \mu \mathrm{Jy}$ in the N2, N3, N4, S7, S9W, S11, L15, L18W, and L24 bands, respectively. The astrometric accuracies of these band detections are $0.48,0.52,0.55,0.99,0.95,1.1,1.2,1.3$, and 1.6 arcsec, respectively. The false-detection rate of all nine bands was decreased to less than $0.3 \%$. In total, 27770 objects are listed in the catalogue, 11349 of which have mid-infrared fluxes.
\end{abstract}

Key words. methods: data analysis - infrared: galaxies - surveys - catalogs

\section{Introduction}

Galaxies in the redshift range $z=1-2$ show significantly more violent activity than their counterparts today. Much of their energy is frequently absorbed by dust and is re-radiated at longer infrared wavelengths, which makes these galaxies luminous in the infrared band. Le Floc'h et al. (2005) have shown that luminous infrared galaxies (LIRGs; $L_{\text {ir }}>10^{11} L_{\odot}$ ) play a major role in galaxy evolution at $z \sim 1$, and Pérez-González et al. (2005) also showed that LIRGs dominate the cosmic

\footnotetext{
* The catalogue is available at the CDS via anonymous ftp to cdsarc.u-strasbg.fr (130.79.128.5) or via http://cdsarc.u-strasbg.fr/viz-bin/qcat?]/A+A/559/A132 or at the ISAS/JAXA observers page, http://www.ir.isas. jaxa. jp/ASTRO-F/Observation/

$\star \star$ Postdoctoral Fellow of the Japan Society for the Promotion of Science.
}

infrared-luminosity density at $z \sim 1-3$. Consequently, the nature of LIRGs is a key objective for advancing our understanding of galaxy evolution. LIRGs are thought to have two main features: they are actively star forming (SF) and host an active galactic nucleus (AGN). Therefore, understanding these features is an important current focus in astrophysics. Although the Spitzer Space Telescope (Werner et al. 2004) is capable of investigating these activities to high sensitivity and spatial resolution, its two main imaging instruments, IRAC (Fazio et al. 2004) and MIPS (Rieke et al. 2004), do not cover the wavelength between 8 to $24 \mu \mathrm{m}$. Hence they cannot detect the redshifted dust features from galaxies in the critical $z=0.5-1.5$ range, such as the emission from polycyclic aromatic hydrocarbon (PAH) feature at $7.7 \mu \mathrm{m}$ or absorption by silicate dust at $9.7 \mu \mathrm{m}$, which are both important diagnostics for SF and AGN activity.

In contrast, the Japanese infrared satellite AKARI provides continuous wavelength coverage over the range from 2 to $24 \mu \mathrm{m}$ 
Table 1. Summary of the IRC properties.

\begin{tabular}{lccccc}
\hline \hline Channel & Band & $\lambda_{\text {ref }}[\mu \mathrm{m}]$ & ${\text { Wavelength }[\mu \mathrm{m}]^{1}}$ & Format[pixel] & Pixel scale[arcsec] \\
\hline \multirow{2}{*}{ NIR } & N2 & 2.4 & $1.9-2.8$ & & \\
& N3 & 3.2 & $2.7-3.8$ & $512 \times 512$ & $1.46 \times 1.46$ \\
& N4 & 4.1 & $3.6-5.3$ & & \\
\hline \multirow{2}{*}{ MIR-S } & S7 & 7.0 & $5.9-8.4$ & & \\
& S9W & 9.0 & $6.7-11.6$ & $256 \times 256$ & $2.34 \times 2.34$ \\
& S11 & 11.0 & $8.5-13.1$ & & \\
\hline \multirow{2}{*}{ MIR-L } & L15 & 15.0 & $12.6-19.4$ & & \\
& L18W & 18.0 & $13.9-25.6$ & $256 \times 256$ & $2.51 \times 2.39$ \\
& L24 & 24.0 & $20.3-36.5$ & & \\
\hline
\end{tabular}

Notes. ${ }^{(1)}$ Defined as the area where the responsivity for a given energy exceeds $1 / e$ of the peak.

in nine overlapping photometric bands, making a deep survey in the direction of the north ecliptic pole (NEP-deep; Matsuhara et al. 2006) using all nine filters. The catalogue from this survey has been created by Wada et al. (2008) and Takagi et al. (2012). The former created single-band catalogues for all nine bands, while the latter created a mid-infrared (MIR) based bandmerged catalogue. In their work, 20000 objects were detected in the near-infrared (NIR) bands, while 7300 objects were detected in the MIR bands. Using these catalogues, Takagi et al. $(2010)^{1}$ found LIRGs with unusually bright emission from PAH, and Goto et al. (2010) evaluated the luminosity function without associated uncertainties from the $K$-correction.

However, the original catalogue leaves room for improvement, especially in the image analysis stage, because most of the images are contaminated by scattered light from inside the detector, stray light from the Earth limb, artificial sources due to bright objects, and patterns from the optical system. The flat-fielding of the 15,18 , and $24 \mu \mathrm{m}$ images also overcorrected the flux of a point source at the edge of the field of view by $\sim 10 \%$ (Arimatsu et al. 2011). By solving these problems, we can improve the catalogue both in the detection limit and in the reliability of the source extraction.

In this paper we devise new image analysis methods and present a new catalogue for the AKARI NEP-deep survey. This paper is organised as follows: In Sect. 2 the NEP survey itself is briefly summarised. In Sect. 3 data reduction using our new methods is described. In Sects. 4.1 and 4.2 source extraction and photometry are described. In Sects. 4.3 to 4.5 , the completeness, the reliability and the astrometric accuracy in the catalogue are evaluated. In Sect. 4.6 optical counterparts are identified. In Sect. 4.7 the catalogue is compared with previous versions. In Sect. 4.8 a basic description of the catalogue contents is given. We summarise our work in Sect. 5.

\section{NEP survey}

The NEP-deep survey covers a $\sim 0.5 \mathrm{deg}^{2}$ circular area located at $\left(\mathrm{RA}=17^{\mathrm{h}} 56^{\mathrm{m}}\right.$, Dec $\left.=66^{\circ} 37^{\prime}\right)$ just offset from the ecliptic pole. The survey was carried out using all nine photometric bands of the Infrared Camera (IRC; Onaka et al. 2007) on board the AKARI satellite (Murakami et al. 2007). Each single band observed $0.6 \mathrm{deg}^{2}$ area, while the area observed with all nine bands was $0.5 \mathrm{deg}^{2}$. The IRC has three channels, NIR, MIR-S, and MIR-L, which work simultaneously with fields of view (FoV) of $10^{\prime} \times 10^{\prime}$. While NIR and MIR-S share the same FoV using a beam splitter, MIR-L has a FoV separated by $20^{\prime}$ from that of NIR/MIR-S. The pixel scales of NIR, MIR-S, and MIR-L are

\footnotetext{
This work was done before the catalogue was published.
}

$1.46 \times 1.46,2.34 \times 2.34$, and $2.51 \times 2.39$ arcsec, respectively. Each channel is equipped with three filters: N2, N3, and N4 in NIR, S7, S9W, and S11 in MIR-S, and L15, L18W, and L24 in MIR-L with the numerals corresponding to the reference wavelength of each filter. The "W" indicates that the filter coverage is wide; S9W covers most of the wavelength range of S7 and S11, while L18W covers most of the wavelength range of L15 and L24. The properties of the IRC are summarised in Table 1.

In the NEP field, additional survey observations are taken with the IRC; the NEP-wide survey, time observations for sensitivity monitoring, performance verification (PV) and ultra-deep observations (UDEEP). The NEP-wide survey covers a larger $5.8 \mathrm{deg}^{2}$ surrounding the NEP-deep field, while the others partially cover the field. The images of the NEP-deep, PV and UDEEP were taken in AOT05 observing mode, in which neither filter changes nor dithering are carried out. On the other hand, the images of NEP-wide and the monitoring observations were taken in the AOT03 observation mode, in which both filter changes and dithering were carried out. These observations were conducted from April 2006 to August 2007.

\section{Revision of image analysis}

The NEP-deep images in the previous work suffer from contamination from scattered light in the detectors, uncertainty in the flat-fielding, stray light from the Earth limb, the so-called soramame pattern originating from the optical system, and artefacts created by bright sources. To remove or reduce these effects, we devised new image analysis methods. The scattered light, stray light, and the patterns in the images were removed by creating templates of their patterns and subtracting them. The artefacts were deleted using their specific characteristics or were simply visually masked out. Furthermore, we added images not from the NEP-deep but from the same region to increase the total number of images covering the field. In addition to this analysis, we also revised the astrometry, mosaicking, and the photometric calibration. On the other hand, general processing such as dark-subtraction and linearity correction were conducted with the standard IRC imaging pipeline ${ }^{2}$. In the following subsection, we describe the revised points of the image analysis in detail.

\subsection{Subtracting scattered light}

In the MIR-S bands, scattered light from the edge of the detector arrays contaminates the images, resulting in a cross-shaped pattern. This effect originates primarily from the sky background, such that all of the images are contaminated by the scattered

2 Version 20110225. The previous work used version 20071017. 


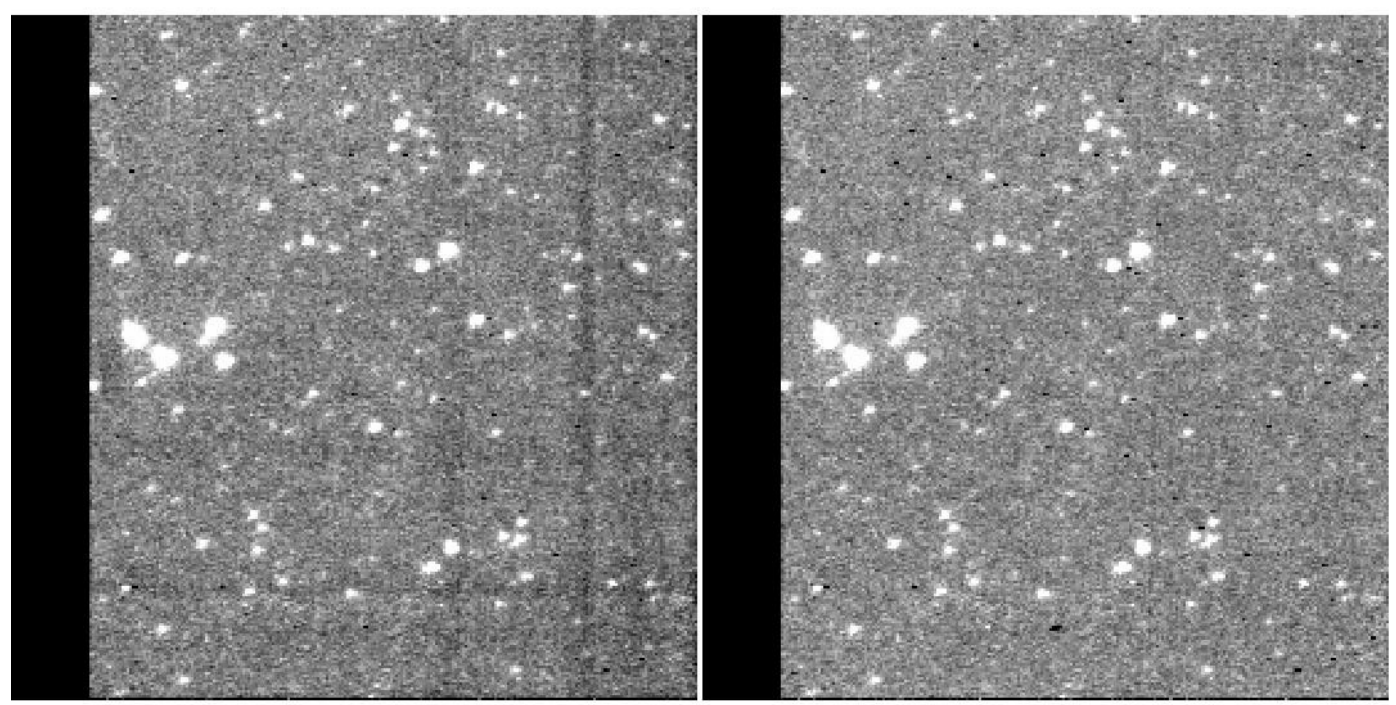

Fig. 1. Subtraction of scattered light in the S9W image. In the previous image (left) the residual of the cross-shaped pattern is seen at the right bottom of the image, whereas the new (right) image shows a much more efficient subtraction.

light. The characteristics of the scattered light in the S7 and S11 bands were investigated by Sakon et al. (2007), but not for S9W. The IRC imaging pipeline used in the previous work applied the simple mean image for the S7 and S11 scattered-light templates as the one for S9W. Even though the wavelength coverage of S9W overlaps those of S7 and S11, this simple mean image is not appropriate for subtracting scattered light in S9W band since the spectrum of the sky background is not uniform.

Therefore, for this study we made a new template for the scattered light in the S9W band. Since the method described in Sakon et al. (2007) is hard to apply for the S9W band because of the small number of available images, we instead took a weighted mean of the templates for S7 and S11, which better accommodates the spectrum of the sky background. Typical values for the sky background in the S7 and S11 bands are 440.639 and 2364.74 ADU (instrumental units). Using these values as weights, we combined the S7 and S11 templates, which were then scaled and subtracted from each S9W image. As a result, the scattered light was subtracted more efficiently (Fig. 1).

On the other hand, in the MIR- $L$ bands the incident light was scattered over the detectors (Arimatsu et al. 2011), and this pattern also appeared in the flat frames used in the pipeline. Hence we used the flat frames as templates for the scattered light, subtracting them from each image.

\subsection{Flat-fielding}

Revised flat frames for each IRC band were created and flatfielding was carried out. The flat frames for the MIR-L in the previous work were affected by scattered light as described above. Arimatsu et al. (2011) subtracted the scattered light and created new flat frames for L15 and L24. Since the wavelength coverage of L18W overlaps those of L15 and L24, in this work the flat frame of L18W was created via a weighted mean of the flats from L15 and L24. The weighting was calculated using the inverse of the conversion factor from ADU to Jy of each band.

In addition, the flat frames of MIR-S were also affected by the so-called soramame (Sect. 3.4) pattern. We revised these flat frames using images that had no such pattern.
Finally, the original flat frames of the NIR channel contained the stray light from the Earth limb (Sect. 3.3). These flats were also revised using images that did not contain any stray light.

\subsection{Subtracting the stray light from the Earth limb}

All images taken from April to August contain stray light from the Earth limb (Fig. 2 left). This light is as strong as the zodiacal light and seriously degrades the image quality in the MIR-S/L images. Since the pattern is not uniform, objects in the valley of the stray light are hardly detectable even if they are as bright as a few hundred micro Jansky. We made templates of the stray-light pattern for each image and subtracted them, noting that when the observing date and the incident angle of the stray-light of the images are similar, the stray-light pattern itself is also similar. To produce these templates, we prepared an image set in which the incident angles and the observing dates agree within 2 degrees and 8 days. The sky background in each image was normalised to unity and the images were combined using the median value. In doing so, we also performed a $3 \sigma$ clip with an upper and lower limit of 1.2 and 0.8 to remove bright objects from the images. After the process, a median-filter box car filter with a three pixel size was applied to the combined images to additionally reduce the photon noise, assuming that the stray light does not fluctuate on such small scales. The resulting templates were then scaled and subtracted from each image. As a result, the stray light was clearly removed (Fig. 2 right). Note that although the pattern can change within a given image set, we were unable to make templates because of the low number of image frames. If many more images were available, that is, other than NEP images, the criteria could be more strict and the templates would improve.

\subsection{Correcting for the SORAMAME pattern}

The MIR-S images taken before 7 January 2007 have a pattern referred to as soramame ${ }^{3}$ at $(X=150: 237, Y=41: 105)$ in detector coordinates. The cause of the pattern is still unknown, but it seems that an obstruction in the optical system affected the image view. Although this affected the pixel values at a level of

This means "broad bean" in Japanese, due to its shape in the image. 

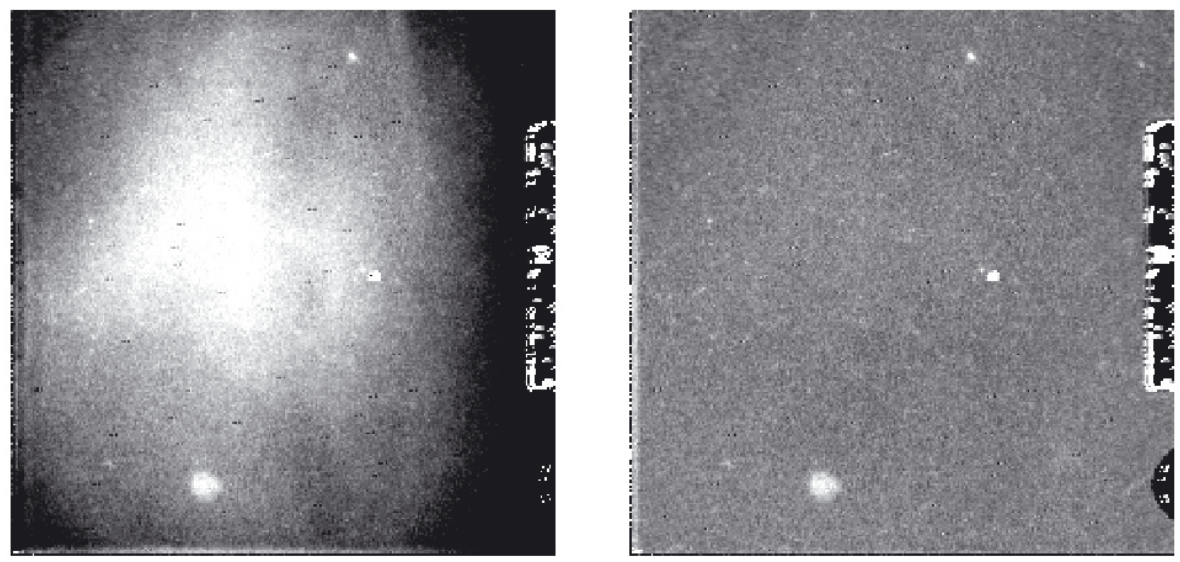

Fig. 2. Subtraction of stray light from the Earth limb. The left image is contaminated by the stray light, which was removed in the right image. The scale of these images is identical.
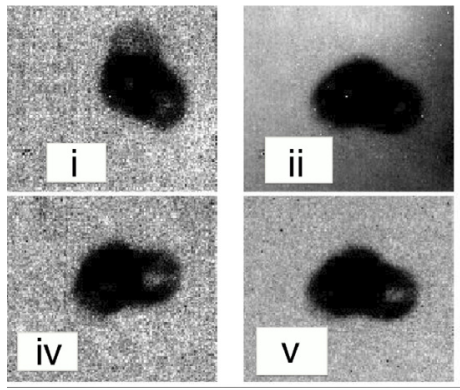

(a)

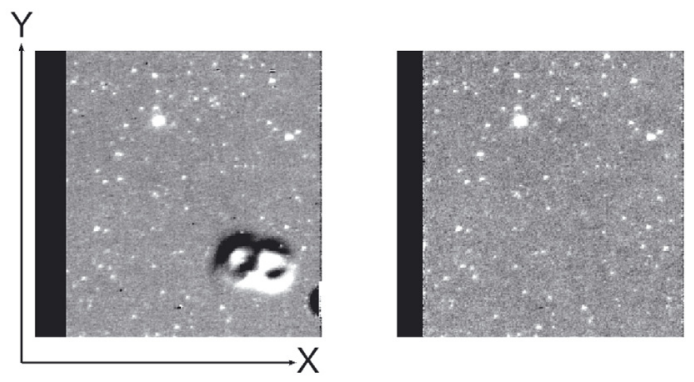

(b)

Fig. 3. a) Five types of the soramame pattern. The pattern (ii) has a gradient in the sky value, showing that it also contains stray light. b) The pattern was corrected for more appropriately using a template of the pattern for each image.

only $\sim 1 \%$, the fluxes could not be measured accurately because the sky background is much brighter than the source signal. However, the pattern can be corrected for in the same manner as the flat-fielding, but with the additional caveat that it has a time dependence (this is why previous efforts were not able to correct it). We found that the pattern can be roughly divided into five periods (Fig. 3a) as follows:
i. $2006 / 4 / 23-2006 / 4 / 26$;
ii. $2006 / 5 / 18-2006 / 10 / 13$;
iii. $2006 / 10 / 15-2006 / 12 / 8$;
iv. $2006 / 12 / 9-2006 / 12 / 14$; and
v. $2006 / 12 / 15-2007 / 1 / 7$.

The blank periods among these five periods arise because there are no images in these periods used for this work.

Even within the same period the pattern changes on a time scale of several days, therefore we prepared the image set in which the observing dates of each image fell within three days.
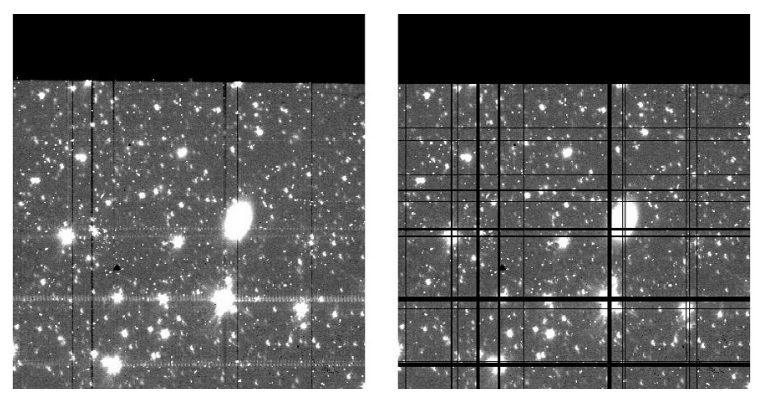

(a)

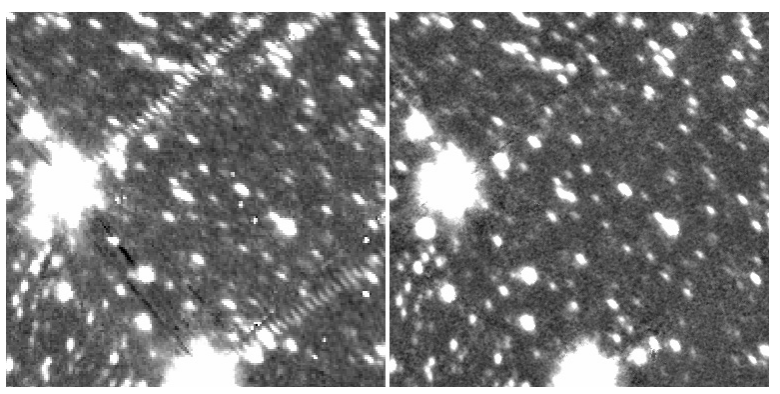

(b)

Fig. 4. a) Muxbleed and column pull-down occur due to bright objects that contaminate the same rows and columns as the objects (left). We masked these rows and columns before mosaicking the images (right). b) As a result, the artefacts disappeared in the mosaicked image (right). We note that the region of panel $\mathbf{a}$ ) is different from that of panel $\mathbf{b})$.

The sky background in each image was normalised to unity and the images were combined with their median value. In doing so, $3 \sigma$ clipping with upper and lower limits of 1.2 and 0.8 was performed after masking all objects in each image. Then the template was scaled and subtracted from each image, and the region ( $X=150: 237, Y=41: 105)$ was divided by the template. As a result, the pattern was corrected for more appropriately than in the previous work (Fig. 3b). We note, however, that the pattern in period (ii) also contains stray light, and in this case the correction was difficult. Similarly as for the stray-light template, we require more images to create more appropriate templates.

\subsection{Removing muxbleed and column pull-down effects}

When bright objects or strong cosmic rays are incident on the NIR band detectors, the images are contaminated by two kinds 

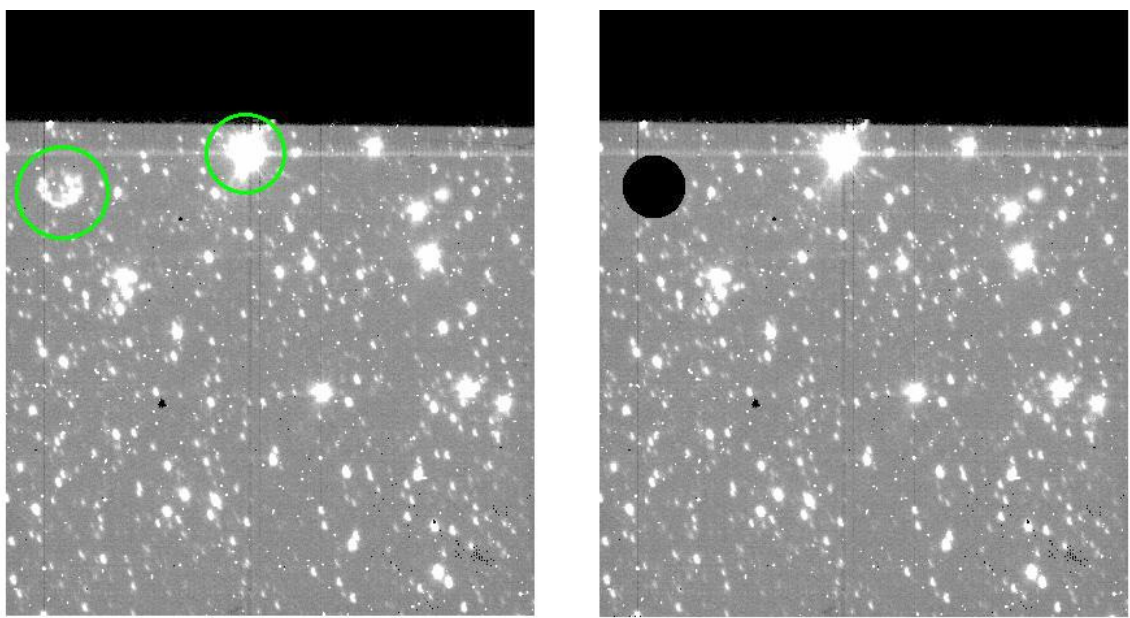

Fig. 5. Artefact in N2 band. It appears in the top left corner and is caused by the bright object in the top centre. Since this artefact is out of focus, it can be identified visually. We masked the artefact with a circle of 36 arcsec radius.

of cross-talk: muxbleed and column pull-down (Fig. 4a left). In particular the former causes many artefacts (Fig. 4b left), since it inserts higher and lower values in turn to pixels in the same row as the pixel with a higher value than some threshold. On the other hand, the column pull-down decreases the pixel value in the same column as the pixel (Lorente et al. 2008). We found this threshold to be $\sim 4000$ ADU for AOT05 and 7000 ADU for AOT03. To overcome these effects, we masked all pixels contaminated by the cross-talk before mosaicking the images (Fig. 4a right). As a result, these contaminations disappeared in the mosaicked images (Fig. 4b right).

On the other hand, effects similar to the column pull-down also appear in MIR-S/L images. However, these arise because bright objects cause the pixel response to temporally degrade. If bright objects are incident on the MIR-S/L detectors during the slow scan of the Far-Infrared Surveyor instrument (FIS; Kawada et al. 2007), the objects were moving along the scan direction in the detector. Since the direction of the slow scan is similar to the column direction, this pull-down effect occurred. We identified this pull-down effect using sigma clipping and masked the columns.

\subsection{Removing artefacts caused by bright objects}

The IRC images have various kinds of artefacts caused by scattered light. When a bright source is incident on the detector in the NIR images, an artefact appears at the symmetrical position with respect to $(X=115, Y=350)$ in the detector coordinate system (Fig. 5). The artefact is out of focus because the optical path is not regular, hence we were able to visually identify the artefact and mask the position with a circle of 36 arcsec radius - larger than the artefact size. Another kind of artefact appears at 55 arcsec to the right of the source position, and another artefact appeared in the 29 arcsec above of the source position in the NIR images. These artefacts are in focus and their brightness is a few percent of that of the source; it is difficult to mask these artefacts because of the high source density in the NIR images. Although this leads to an increase in fake sources, it does not affect the results because only objects with optical counterparts are entered in our catalogue (see also Sect. 4.1). One may be led to think that these artefacts cause underestimates in source fluxes, but because all sources have these artefacts, and even the flux calibration contains these effects, these artefacts do not affect the flux measurements.

In MIR-S images an artefact appears at 55 arcsec above the source position (Arimatsu et al. 2011, see also their Fig. 1), with a brightness of a few percent of that of the source. We masked this position with a circle of 18 arcsec radius for sources brighter than 14 mag in each band. This threshold was chosen such that the brightness of the artefacts is negligible. Note that although secondary and tertiary artefacts also exist, they are too faint to be considered.

Finally, MIR-L images have other kinds of artefacts (Arimatsu et al. 2011, see also their Fig. 3). Since these artefacts are much brighter, we did not use the images with these artefacts. Note that such artefacts only appear around NGC 6543 (the Cat's Eye nebula).

\subsection{Addition of images}

To increase the number of images, we used not only the NEPdeep images, but also other observations around the NEP: the NEP-wide survey, time observations for sensitivity monitoring, performance verification and ultra-deep observations. To avoid affecting the uniformity of the survey depth, all images of NEPwide and some images of the others were added. These images were processed with the same methods as the NEP-deep images. As a result, the total number of individual images was increased by $\sim 15 \%$, leading to an improvement in the survey depth of up to $7 \%$.

\subsection{Associations}

After the processes described above, sky subtraction, bad-pixelmasking and trimming of bad parts in the images were performed. To subtract the sky background, median-filtered images with a box size of 31 pixels, which is larger than the point spread function (PSF), were created after masking the bright objects using a $3 \sigma$ clipping technique. In this process we measured the sky background without contamination from bright objects. Then the median-filtered images were subtracted from each image.

For the bad pixel mask, we used dark frames from observations conducted within several days of the NEP images. Typically, 100 dark images were stacked and an area of around 15 pixels around each pixel in the stacked dark frame was used to calculate the median value and normalised median absolute deviation (Beers et al. 1990).

$$
\begin{aligned}
& \left\|p_{i}-\operatorname{med}\left\{p_{i}\right\}\right\|>3 \sigma \\
& \sigma=1.4826 \operatorname{med}\left\{\left\|p_{i}-\operatorname{med}\left\{p_{i}\right\}\right\|\right\}
\end{aligned}
$$


where $p_{i}$ indicates the pixel values and med indicates the median value. If the pixel value deviated more than $3 \sigma$ from the median value, the pixel was regarded as a bad pixel and was masked.

We trimmed the edge and the masked region of each image because these regions might not have a regular response, especially in images contaminated by stray light. The trimmed regions were $[X=1: 412, Y=381: 512]$ in NIR, $[X=1: 32, Y=$ 1:256] and three pixels at the edges of MIR-S, $[X=237: 256$, $Y=1: 256]$ and three pixels at the edges of MIR-L images.

\subsection{Astrometry}

In the previous work, world coordinate system (WCS) information was derived after stacking the images in each individual pointing observation using the standard pipeline. This led to an increase in the amount of pixelation, that is, for distortion correction, alignment images for the first stacking and mosaicking in the next step. Since pixelation would corrupt the images, it is best to reduce the number of instances of this process. Consequently, mosaicked images should be created with only one degree of pixelation. Therefore, in this work, the WCS was attached to each image in each individual observation.

We used the 2-Micron All Sky Survey (2MASS; Skrutskie et al. 2006) as a reference catalogue for the astrometry in the N2, N3, N4, S7, and S9W images. For the S11 and L15 bands, the S9W catalogue was used, and for the L18W and L24 bands, the L15 catalogue was used since only a few objects in these bands have counterparts in the 2MASS catalogue.

In adding WCS information to the N2, N3 and N4 images, we used the PUTWCS task in the IRC pipeline, in which source extraction and a cross-match with 2MASS catalogue are automatically performed. For the longer wavelength bands we adopted following method:

1.) we reduced the pixel size to half the size to improve the precision of the cross-match;

2.) we stacked all images in each observation;

3.) we cross-matched with the $2 \mathrm{MASS}, \mathrm{S} 9 \mathrm{~W}$ or L15 catalogue to register the WCS information carefully, and all of the WCS information was visually confirmed;

4.) we copied the WCS information on the original images in each observation.

By this process, the WCS information was registered on the images without pixelation. We note that the image distortion correction was included in this calibration.

\subsection{Mosaicking}

Before mosaicking the images, we masked pixels that had outlier values using a method similar to that implemented in the A WISE Outlier Detector (AWOD; Masci \& Fowler 2009) with 4-sigma clipping, where sigma is defined by Eq. (2). This is because the outlier value can affect the interpolation of the pixel value in the next step.

To create mosaicked images, we projected all images onto one common image using the WCS information and combined them. In projecting the images, pixelation with linear interpolation was carried out, without using pixels that differed by more than 0.7 pixels from the original position. With this process we reduced the pixel size from $1.46,2.34$, and 2.45 arcsec to 0.7 , 1.0 , and 1.0 arcsec for the NIR, MIR- $S$, and MIR- $L$ bands. After the projection, all the images were stacked by taking an average value with 4 -sigma clipping. To improve the reliability of source
Table 2. List of the standard stars used for the photometric calibration.

\begin{tabular}{lccc}
\hline \hline Name & RA & Dec & $K$ s mag \\
\hline 1757132 & 269.305190 & 67.061363 & 11.155 \\
KF03T1 & 269.433111 & 66.448715 & 9.923 \\
KF03T2 & 269.464464 & 66.517632 & 8.963 \\
KF06T2 & 269.658286 & 66.781174 & 11.149 \\
KF03T3 & 269.754790 & 66.557289 & 10.925 \\
KF03T4 & 269.766472 & 66.516495 & 10.091 \\
KF09T1 & 269.846003 & 66.048943 & 8.114 \\
KF06T4 & 269.858598 & 66.916161 & 11.240 \\
KF01T4 & 271.013118 & 66.912766 & 8.067 \\
KF01T5 & 271.016205 & 66.928833 & 11.072 \\
Bp66_1073 & 270.789993 & 66.469994 & 7.544 \\
\hline \multicolumn{4}{c}{ Only MIR-L } \\
HD42525 & 91.539091 & -66.039622 & 5.751 \\
HD 166780 & 272.161750 & 57.979667 & 3.963 \\
HD 158485 & 261.520167 & 58.651917 & 6.145 \\
NPM1p67_0536 & 269.727750 & 67.793556 & 6.409 \\
NPM1p65_0451 & 253.404333 & 65.638194 & 6.524 \\
Bp66_1060 & 269.000771 & 66.928619 & 6.720 \\
\hline
\end{tabular}

extractions and photometry, we prepared two mosaicked images using different image sets among which the images were divided according to two observation spans so that each pixel in the two images had the same number of images (see also Sect. 4.1), hereafter referred to as the $\mathrm{A}$ and $\mathrm{B}$ images, and the mosaicked image of the whole data set, the $\mathrm{A}+\mathrm{B}$ image. In addition, we made noise and coverage images of them, with pixel values containing the standard deviation of the pixel values and the number of images used to combine them.

\subsection{Photometric calibration}

Although the photometric calibration has been estimated by Tanabé et al. (2008) using standard stars, we performed our own photometric calibration for our analysis, since the standard calibration factor would have changed because the PSF in images is pixelated through the IRC imaging pipeline and differs from ours.

To obtain the calibration factor from ADU to $\mu \mathrm{Jy}$, we compared the flux densities of standard stars with their observed value in terms of ADU. The flux densities of the standard stars were calculated by Eq. (4) in Tanabé et al. (2008),

$f_{\lambda i}^{\text {quoted }}=\frac{\int_{\lambda i_{s}}^{\lambda i_{e}} R_{i}(\lambda) \lambda f_{\lambda}(\lambda) \mathrm{d} \lambda}{\lambda_{i} \int_{\lambda i_{s}}^{\lambda i_{e}} R_{i}(\lambda) \mathrm{d} \lambda}$,

where $f_{\lambda}$ is taken from the Cohen templates (Cohen et al. 1996, 1999, 2003a,b; Cohen 2003), $R_{i}$ is the spectral response of each IRC band ${ }^{4}$, and $\lambda_{i s}$ and $\lambda_{i e}$ are the wavelengths at which $R_{i}$ becomes zero. Since most of the standard stars are in the NEP field, we only used one in this field for the NIR and MIR- $S$ bands. For the MIR- $L$ bands these stars are too faint, hence we also used other standard stars. The stars we used for this calibration are listed in Table 2. The images of the standard stars were analysed with the same method as the NEP-deep images. Some objects were not used because of saturation or contamination from nearby bright objects. Aperture photometry was carried out for several aperture radii. Then we compared the ADU values from the aperture photometry to the flux densities of the standard stars

4 http://www.ir.isas.jaxa.jp/ASTRO-F/Observation/ RSRF/IRC_FAD/, unit: electronperenergy 
Table 3. Conversion factors from ADU to $\mu \mathrm{Jy}$ for each band.

\begin{tabular}{lcc}
\hline \hline Band & $\begin{array}{c}\text { Conversion } \\
\text { factor[ } \mu \text { Jy/ADU] }\end{array}$ & Err[\%] \\
\hline N2 & 0.328 & 1.4 \\
N3 & 0.277 & 1.2 \\
N4 & 0.214 & 2.0 \\
S7 & 1.254 & 0.9 \\
S9W & 0.740 & 1.0 \\
S11 & 1.044 & 0.6 \\
L15 & 2.532 & 1.5 \\
L18W & 1.974 & 2.2 \\
L24 & 9.134 & 3.6 \\
\hline
\end{tabular}

Notes. The aperture radii are 9 and 6 pix for NIR and MIR-S/L. These correspond to $6.3^{\prime \prime}$ and $6.0^{\prime \prime}$.

and obtained the conversion factors for each aperture photometry. Table 3 shows the conversion factors at 6.3 and 6.0 arcsec aperture radii for NIR and MIR-S/L. These sizes are similar to or slightly larger than the PSF size. We note that the flux calibration is based on only about ten objects so that calibration errors may be underestimated.

\section{Catalogue}

\subsection{Source detection}

Different algorithms for source detection in the MIR and NIR bands were used because the detections are limited by different effects. Because the detection from the MIR bands is limited by the sky noise, we created detection images by stacking all six bands, to significantly reduce the sky noise. On the other hand, the detection from the NIR bands is limited by source confusion, therefore source extraction was conducted directly from each band image.

To create the detection images for the MIR source extraction, we stacked all six MIR bands with a $\chi^{2}$ method, similar to the procedure described by Szalay et al. (1999). In this process, the pixel values of each image were converted into a $\chi^{2}$ value using noise and coverage images of each mosaicked image created in Sect. 3.10,

$\chi_{i}^{2}=\left|S_{i} / \sigma_{\mu i}\right|\left(S_{i} / \sigma_{\mu i}\right)$

$\sigma_{\mu i}=\sigma_{i} / \sqrt{N_{i}}$

$i=a 1, a 2, \ldots, a 6, b 1, \ldots, b 6$,

where $S_{i}$ is the pixel value of the mosaicked images, $\sigma_{i}$ and $N_{i}$ are those of the noise and coverage images, and $i$ indicates the A and B images of each MIR band from S7 to L24. Note that the definition of the $\chi^{2}$ is not standard to account for the conservation of the sign of the value, that is, no real object should be on the pixels with negative value. In the stacking process, we clipped pixels in which the values were lower than -9 (which corresponds to $-3 \sigma$ ), or the number of images used for mosaicking were lower than 15 , to remove any outlier values. In this process we also made A and B images, that is, we stacked the a1 to $\mathrm{a} 6$ and $\mathrm{b} 1$ to b6 images separately.

Using the detection images, we took the following method to improve the reliability of the source extraction. We first made reference samples and then took objects to be real sources when they were selected from the reference sample and also detected in at least one of the single-band mosaicked images. To construct the reference sample we detected sources from the A, B
Table 4. SExtractor parameters.

\begin{tabular}{|c|c|c|c|c|}
\hline PARAM & A,B & $\mathrm{A}+\mathrm{B}$ & Single & NIR \\
\hline DETECT_THRESH & $3(2.5)$ & $4(3)$ & $2.0(2.0)$ & 3.0 \\
\hline DETECT_MINAREA & $12(6)$ & $8(6)$ & $15(10)$ & 12 \\
\hline WEIGHT_TYPE & \multicolumn{2}{|c|}{ NONE } & \multicolumn{2}{|c|}{ MAP_RMS } \\
\hline WEIGHT_IMAGE & \multicolumn{2}{|c|}{ - } & \multicolumn{2}{|c|}{ rms image } \\
\hline BACK_TȲPE & \multicolumn{2}{|c|}{ MANUAL } & \multicolumn{2}{|c|}{ AUTO } \\
\hline BACK_VALUE & \multicolumn{2}{|c|}{0} & \multicolumn{2}{|c|}{ - } \\
\hline CLEAN & $\mathrm{N}$ & $\mathrm{N}$ & $\mathrm{N}$ & Y \\
\hline DEBLEND_MINCONT & 0 & 0 & 0 & 0 \\
\hline BACK_SIZE & - & - & 256 & 256 \\
\hline BACK_FILTERSIZE & - & - & 6 & 3 \\
\hline MASK_TYPE & \multicolumn{4}{|c|}{ CORRECT } \\
\hline BACKP̈HOTO_TYPE & \multicolumn{4}{|c|}{ GLOBAL } \\
\hline FILTER_NAME & \multicolumn{4}{|c|}{ gauss_3.0_7 7 7.conv } \\
\hline
\end{tabular}

Notes. Values in round brackets are for the initial detection to create the reference sample.

and $\mathrm{A}+\mathrm{B} \chi^{2}$ images and single-band images using SExtractor (Bertin \& Arnouts 1996) twice with the different parameter sets listed in Table 4. First, the parameters were set to be initial estimates (in round brackets in Table 4). Then we compared the objects from the $\chi^{2}$ and the single-band images. In this process 8676 objects were detected at the same position within 1.5 arcsec in all $\chi^{2}$ images and in at least two single-band images. In addition, 178 objects were detected in all $\chi^{2}$ and the three singleband images at the same position within 2.5 arcsec. These objects were considered to be real and were listed as reference samples. Secondly, the parameters were more tightly constrained (without round brackets in Table 4) and all objects detected in all $\chi^{2}$ images at the same position within 2.5 arcsec were listed as the reference sample. In total, 9701 sources were obtained as references. We then extracted sources from each single-band image and compared them with the references. When a source was detected at the same position to within 2.5 arcsec from the reference, it was considered to be real. We note that a region centred at $(\mathrm{RA}=269.60858, \mathrm{Dec}=66.62320)$ with the radius of 5 arcmin was not used to avoid the scattered light from NGC 6543 (Cat's Eye nebula), because it creates many artificial sources. For detection from the single-band images, we used weight images whose pixel values were the rms values created from the noise images divided by the square root of the coverage images.

The total number of objects detected in each band are listed in Table 5. More than 6000 objects were detected in each singleMIR band, except for the L24 band. Since the sensitivity of L24 band is relatively low, the number of detected objects is just above 3000. Table 6 shows the number of objects detected in multiple bands. It also shows that the number of objects detected with at least one band is 9560, an increase of 2000 compared to the previous work.

For the source extraction from the NIR images, we did not use any detection image since the detection is limited by source confusion, and stacking the three NIR bands can hardly improve the detection limit. Instead, to improve the reliability of the source detection, we entered only objects with optical counterparts in our catalogue. For optical catalogues, we used the catalogue based on CFHT/MegaCam $z^{\prime}$ band (Oi et al., in prep.), the WIRCam $K$ s band, and the Subaru/S-cam $z^{\prime}$ band (see also Sect. 4.6). The detection limits of these catalogues are $\sim 24,23$ and $26 \mathrm{AB}$ mag, respectively. Compared with the N2 band, the WIRCam Ks-band catalogue has almost the same 
Table 5. Effective area, the number of detected sources in the positive and negative images, and the $5 \sigma$ limit for each band.

\begin{tabular}{lcccc}
\hline \hline Band & Area $\left[\mathrm{min}^{2}\right]$ & Number & Fake $(\%)$ & $S_{\text {lim }}=5 \sigma[\mu \mathrm{Jy}]$ \\
\hline N2 & 2069 & 17108 & $5(0.03)$ & 11.5 \\
N3 & 2083 & 19933 & $3(0.02)$ & 9.0 \\
N4 & 2053 & 18671 & $0(0)$ & 10.4 \\
S7 & 2100 & 6307 & $16(0.25)$ & 29.7 \\
S9W & 2068 & 7059 & $18(0.25)$ & 33.6 \\
S11 & 2082 & 6005 & $13(0.22)$ & 56.5 \\
L15 & 2143 & 6233 & $10(0.16)$ & 86.8 \\
L18W & 2149 & 6526 & $9(0.14)$ & 93.1 \\
L24 & 2186 & 3278 & $7(0.21)$ & 255.6 \\
\hline
\end{tabular}

Table 6. Cumulative number of objects detected in at least $N_{\text {Band }}$ bands.

\begin{tabular}{ccc}
\hline \hline$N_{\text {Band }}$ & Number & Fake $(\%)$ \\
\hline 1 & 9560 & $45(0.47)$ \\
2 & 8975 & $19(0.21)$ \\
3 & 6948 & $8(0.12)$ \\
4 & 4742 & $1(0.02)$ \\
5 & 3355 & $0(0.00)$ \\
6 & 1823 & $0(0.00)$ \\
\hline
\end{tabular}

wavelength coverage, but is $\sim 2$ mag deeper. We detected sources in each NIR band and compared them with the optical catalogues using a search radius of 2.1 arcsec, slightly larger than the $3 \sigma$ positional accuracy. Furthermore, we removed any objects with an $S / N<2$ (see also Sect. 4.2) since they probably are fake sources. The parameters of SExtractor are also listed in Table 4 and the number of detected objects is listed in Table 5. It shows that the number of the sources is lower than 20000 for the entire NIR bands, a decrease from the previous work. This is because the detection criteria are stricter than the previous work to improve the reliability. Moreover, because of the detection limit and/or areal coverage of these optical catalogues some objects were excluded. However, these objects have a minor impact on the results, as described in Sect. 4.7.

\subsection{Photometry}

We performed aperture photometry on the $\mathrm{A}+\mathrm{B}$ images with aperture radii of 6.0 and 6.3 arcsec for MIR and NIR bands using SExtractor. Aperture correction was not needed because it had been calculated in the conversion factor for each aperture radius.

Photometric errors were also estimated using A and B images by measuring the fluxes at the same position in the $\mathrm{A}$ and $\mathrm{B}$ images. We assumed that the error distribution of the fluxes from the A and B images are the same and used the relation between the expected difference of two samples and the standard deviation,

$$
\begin{aligned}
\langle\|\Delta\|\rangle & =\sqrt{2 / \pi} \times \sigma_{\mathrm{A}-\mathrm{B}} \\
& =\sqrt{2 / \pi} \times \sigma_{\mathrm{A}, \mathrm{B}} \times \sqrt{2},
\end{aligned}
$$

where $\Delta$ is the difference of fluxes from $\mathrm{A}$ and $\mathrm{B}$ images, and $\sigma_{\mathrm{A}-\mathrm{B}}=\sigma_{\mathrm{A}, \mathrm{B}} \times \sqrt{2}$. Since the average error is expressed as $\sigma_{\text {avg }}=$ $\sigma_{\mathrm{A}, \mathrm{B}} / \sqrt{2}$, the standard deviation of the fluxes in the $\mathrm{A}+\mathrm{B}$ image
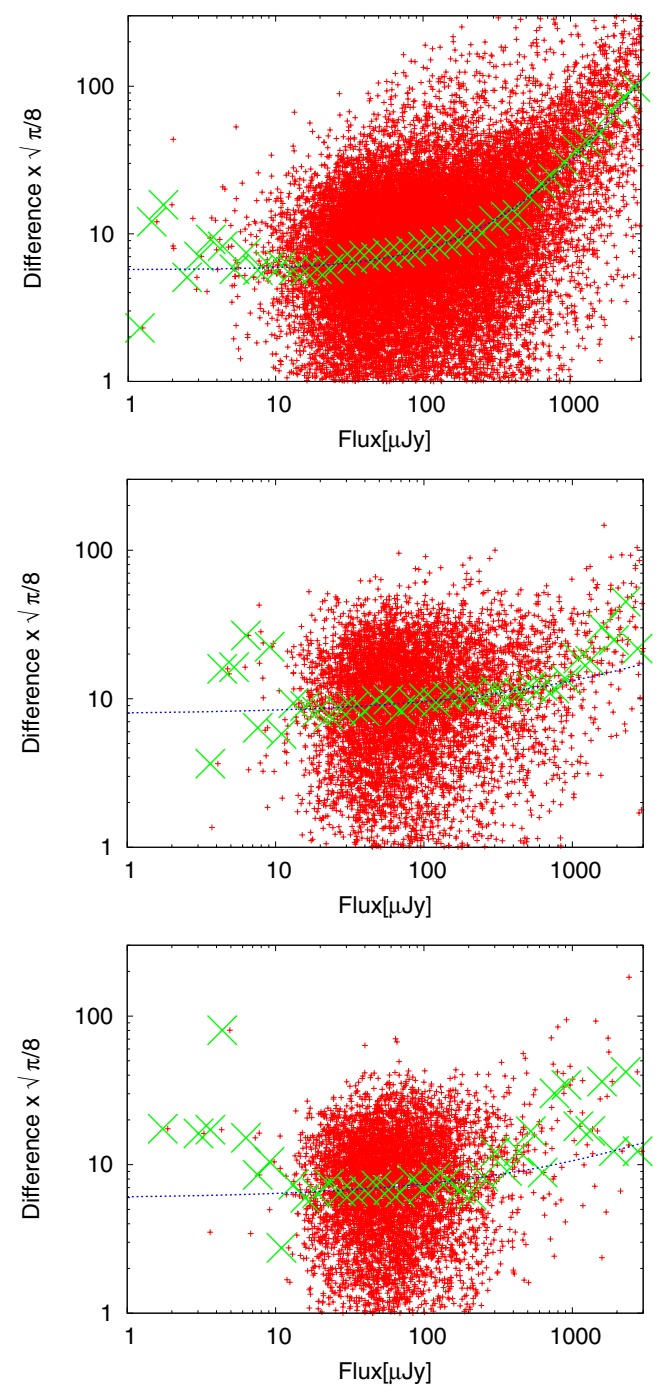

Fig. 6. Flux errors are estimated from the difference between the fluxes from the A and the B images. In the top, middle, and bottom panels, flux errors in N3, S9W, and L15 are shown. Red points indicate the difference multiplied by $\sqrt{\pi / 8}$. Green crosses indicate the median value in a log flux bin size of 0.08 . The blue dotted line shows the fitting result. The higher value of the red point and the line was adopted as the flux error.

can be written as

$\sigma_{\mathrm{A}+\mathrm{B}}=\sqrt{\pi / 8} \times\|\Delta\|$.

To express these values as a function of the fluxes, we took the median of these values in a log flux bin size of 0.08 and fitted them with the following functions (Fig. 6):

$\Delta f \times \sqrt{\pi / 8}=a \times f l u x^{2}+b \times f l u x+c \quad$ for NIR

$\Delta f \times \sqrt{\pi / 8}=a \times \sqrt{f l u x}+b \quad$ for MIR.

The higher value of the difference multiplied by $\sqrt{\pi / 8}$ and the fitting result was adopted as the flux error. The detection limit for each band was calculated from the fitting results and is listed in the last column of Table 5.

One might think PSF photometry to be superior to our method, especially in blended sources. However, since the positional precision is only moderate in our catalogue (1-2 arcsec compared with PSF size, 5-7 arcsec), the centring of the PSF fitting is difficult, hence we adopted aperture photometry as our preferred method. 
K. Murata et al.: Revised catalogue of AKARI NEP-Deep survey

Table 7. Completeness as a function of flux density in each band in percent.

\begin{tabular}{lccccccccc}
\hline \hline Flux[ $\mu \mathrm{Jy}]$ & $\mathrm{N} 2$ & $\mathrm{~N} 3$ & $\mathrm{~N} 4$ & S7 & S9W & S11 & L15 & L18W & L24 \\
\hline 10.96 & 2.9 & 4.7 & 7.0 & 1.8 & 1.2 & 0.2 & 0.0 & 0.0 & 0.0 \\
13.18 & 4.7 & 8.1 & 12.4 & 5.7 & 3.6 & 0.3 & 0.0 & 0.0 & 0.0 \\
15.85 & 9.0 & 13.3 & 20.7 & 11.3 & 8.3 & 0.9 & 0.0 & 0.0 & 0.0 \\
19.05 & 15.5 & 19.1 & 28.8 & 21.7 & 16.4 & 2.1 & 0.2 & 0.0 & 0.0 \\
22.91 & 21.1 & 22.1 & 35.0 & 34.8 & 27.3 & 5.2 & 0.1 & 0.2 & 0.0 \\
27.54 & 27.7 & 29.6 & 41.3 & 47.1 & 38.6 & 12.0 & 0.5 & 0.5 & 0.0 \\
33.11 & 33.6 & 34.7 & 46.5 & 55.6 & 49.1 & 20.9 & 1.7 & 1.5 & 0.0 \\
39.81 & 38.4 & 41.4 & 51.0 & 63.9 & 57.9 & 33.7 & 4.4 & 4.2 & 0.0 \\
47.86 & 43.7 & 46.1 & 57.3 & 73.5 & 67.2 & 47.3 & 10.6 & 9.5 & 0.0 \\
57.54 & 51.4 & 51.5 & 64.0 & 80.5 & 74.4 & 56.7 & 21.8 & 17.7 & 0.0 \\
69.18 & 56.8 & 56.4 & 67.8 & 85.0 & 80.6 & 67.4 & 34.6 & 29.8 & 0.1 \\
83.18 & 59.4 & 60.8 & 71.1 & 88.0 & 84.5 & 74.9 & 47.5 & 42.4 & 0.5 \\
100.00 & 65.0 & 67.0 & 75.8 & 91.1 & 88.2 & 81.4 & 60.0 & 53.3 & 2.2 \\
120.23 & 67.7 & 72.0 & 80.3 & 93.5 & 90.8 & 85.8 & 67.1 & 62.2 & 5.3 \\
144.54 & 73.0 & 75.0 & 84.2 & 94.2 & 92.3 & 88.3 & 76.2 & 70.4 & 11.7 \\
173.78 & 77.0 & 79.7 & 86.6 & 94.6 & 93.5 & 91.4 & 82.6 & 77.0 & 24.4 \\
208.93 & 78.2 & 82.8 & 88.6 & 95.4 & 94.5 & 93.4 & 85.7 & 83.6 & 37.9 \\
251.19 & 81.7 & 85.5 & 91.0 & 96.1 & 95.6 & 94.0 & 89.4 & 87.8 & 51.2 \\
302.00 & 83.8 & 86.9 & 92.2 & 96.7 & 95.9 & 94.9 & 91.8 & 90.8 & 63.1 \\
363.08 & 86.5 & 88.2 & 93.7 & 96.8 & 96.3 & 96.3 & 94.7 & 92.5 & 74.7 \\
436.52 & 89.1 & 90.3 & 94.9 & 97.5 & 96.5 & 96.6 & 95.1 & 94.2 & 82.6 \\
524.81 & 89.6 & 92.5 & 96.0 & 97.6 & 96.9 & 96.9 & 96.2 & 95.3 & 88.1 \\
630.96 & 90.8 & 93.3 & 95.9 & 98.1 & 97.6 & 97.3 & 96.4 & 95.6 & 90.9 \\
758.58 & 91.6 & 94.2 & 95.8 & 98.2 & 98.2 & 97.7 & 97.4 & 96.3 & 93.2 \\
912.01 & 93.3 & 94.2 & 96.5 & 98.5 & 98.4 & 98.0 & 97.3 & 96.9 & 95.8 \\
1096.48 & 94.4 & 95.5 & 97.1 & 98.6 & 98.5 & 98.3 & 97.5 & 97.2 & 95.8 \\
1318.26 & 95.3 & 95.5 & 97.5 & 98.6 & 98.4 & 98.5 & 98.2 & 97.7 & 96.9 \\
1584.89 & 94.9 & 96.0 & 97.6 & 98.6 & 98.0 & 98.9 & 98.1 & 97.7 & 97.2 \\
1905.46 & 95.8 & 96.7 & 97.2 & 98.7 & 98.3 & 98.9 & 97.5 & 98.0 & 97.5 \\
2290.87 & 95.8 & 97.3 & 98.0 & 98.9 & 98.8 & 98.9 & 97.4 & 98.4 & 97.7 \\
2754.23 & 96.1 & 97.4 & 98.1 & 99.0 & 98.8 & 98.7 & 98.1 & 98.0 & 97.6 \\
3311.31 & 96.8 & 97.4 & 98.1 & 99.2 & 98.8 & 98.7 & 97.7 & 97.8 & 98.2 \\
\hline & & & & & & & & &
\end{tabular}

Notes. The flux bin size is $0.2 \mathrm{mag}$.

\subsection{Completeness}

We calculated the completeness level of our detections via simulations using artificial sources. We injected artificial sources with the same radial profile as the PSF into our images and extracted them using SExtractor with the same parameters. The input artificial sources were separated by more than 60 pixels from each other to avoid self-confusion. The PSFs were investigated by Arimatsu et al. (2011) for S7, S11, L15 and L24 bands. For other bands we created simple PSFs using point sources or the weighted average of the above PSFs. We regarded the source as successfully extracted when the position of the extracted source agreed within 2.5 and 2.1 arcsec for the MIR and NIR bands, respectively, the same criteria as for the matching with $\chi^{2}$ or ground-based catalogues, and the magnitude agreed within $0.5 \mathrm{mag}$, the same as Wada et al. (2008). We defined the completeness as the ratio of the number of extracted sources to the number of input sources. The calculation was performed at each magnitude with an interval of 0.2 mag. For the MIR bands we injected 100 sources per simulation, and repeated this 20 times. On the other hand, for the NIR bands, considering the source density, we injected only 20 sources per simulation and repeated the simulation 100 times. The results are summarised in Table 7.

Figure 7 shows the source count obtained from this work with red solid lines, while the previous ones (from Takagi et al.) are shown as green solid lines. In the top, middle, and bottom panels, those of S9W, S11 and L15 bands are compared.
The L15 source counts in the previous work are distributed at $\sim 10 \%$ to brighter flux densities than ours. This might result from the different flux calibration or from the flat-fielding (see also Sect. 4.7). In our source counts, the peak in the counts in S9W, S11, and L15 bands occur at 40, 57, and $120 \mu \mathrm{Jy}$, whereas the previous work reported the peaks at 58, 69, and $160 \mu \mathrm{Jy}$. This indicates that the detection limits in our catalogue are $\sim 20 \%$ deeper than the previous ones in all bands. Although the previous work has evaluated the completeness of their catalogue, we could not compare this with ours because the previous completeness calculation was evaluated from detection images only, and does not reflect the completeness of the single-band detection.

We compared our results with the Wide-field Infrared Survey Explorer (WISE; Wright et al. 2010) NEP catalogue, which has four overlapping bands at 3.4, 4.6, 12, and $22 \mu \mathrm{m}$ (Jarrett et al. 2011). This showed that source counts of these bands peak at $41,30,166$, and $1355 \mu \mathrm{Jy}$. On the other hand, our source counts of the N3, N4, S11, and L24 bands peak at 13, 11, 57, and 302, much deeper than those of WISE.

For the NIR catalogue we used only objects that matched the ground-based catalogue, which might introduce a bias against the reddest and faintest sources. We estimated to which degree the completeness could be reduced in the worst case, assuming all objects to be mismatched with ground-based catalogues to be real. We found that these effects were only 2-3\% at a $50 \%$ completeness limit of the N2, N3, and N4 bands, which are 56, 55, and $38 \mu \mathrm{Jy}$. However, the completeness at brighter than $1000 \mu \mathrm{Jy}$ was reduced by more than $20 \%$. The reason was 

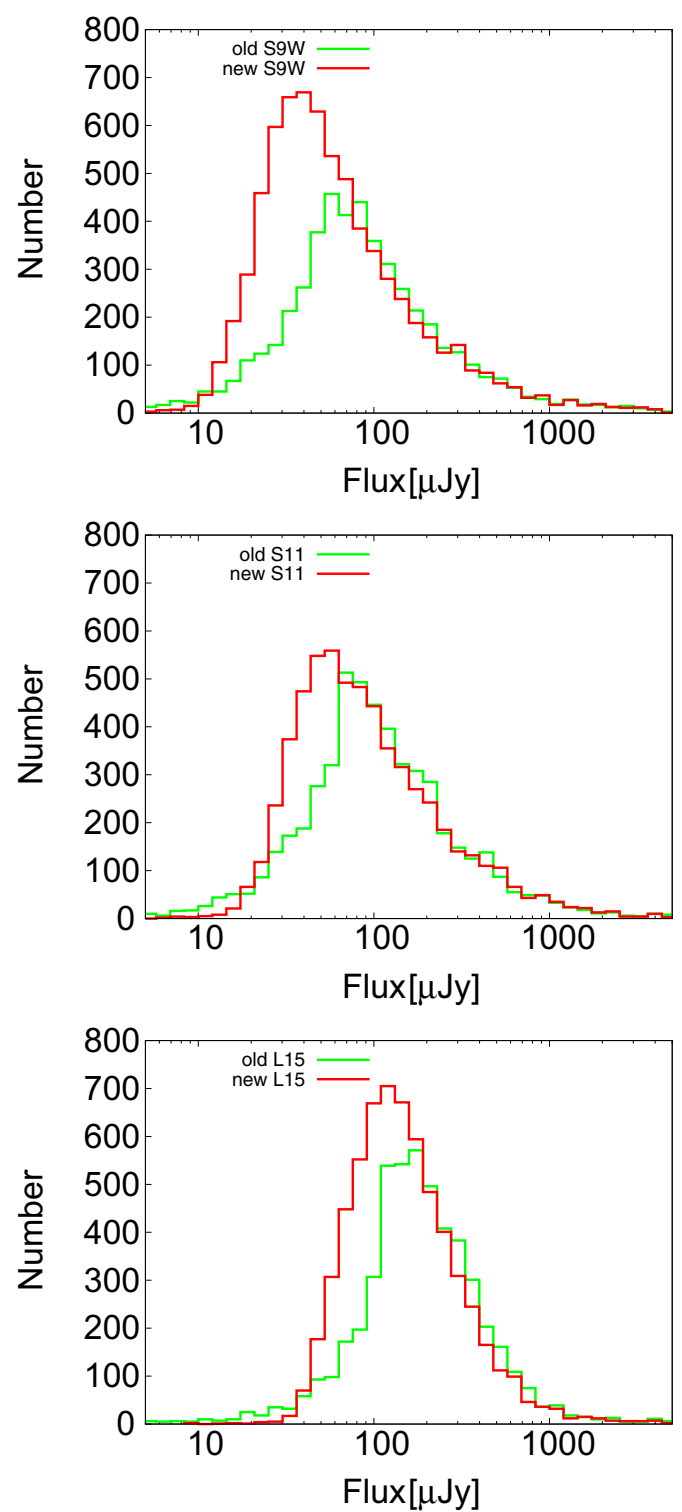

Fig. 7. Number of sources detected in the S9W, S11, and L15 bands. Green lines indicate previous results, red lines indicate this work.

that these objects were saturated in the ground-based catalogue and were not listed (see Oi et al., in prep., for details). Therefore we concluded that the completeness at fainter than $1000 \mu \mathrm{Jy}$ was not reduced significantly by this effect.

\subsection{Reliability of the source detection}

To determine the reliability of the source detection, we estimated the false-detection rate using the negative images. The negative images are almost identical to the positive images multiplied by -1 , but we masked the pixels with values higher than 9 in the same way as the positive images. If the false detection were caused only by sky noise, the number of fake sources is expected to be the same in both positive and negative images. We performed source extraction on the negative images with the same method as for the positive images. The estimated number of the false detections are also summarised in Table 5. The estimated false-detection rate in all nine bands is lower than $0.3 \%$. Therefore we conclude that the single-band detection in our catalogue is reliable at $99.7 \%$.
Table 8. Positional accuracy of the N2-L24 band.

\begin{tabular}{lccccc}
\hline \hline & \multicolumn{2}{c}{ RA[arcsec] } & \multicolumn{2}{c}{ Dec[arcsec] } & \\
Band & Mean & rms & Mean & rms & Total \\
\hline N2 & 0.00178 & 0.32928 & -0.046154 & 0.34887 & 0.4797 \\
N3 & -0.01869 & 0.34988 & -0.057003 & 0.37982 & 0.5164 \\
N4 & -0.01438 & 0.37579 & -0.068680 & 0.4029 & 0.5510 \\
S7 & -0.01615 & 0.68214 & -0.18314 & 0.72016 & 0.9919 \\
S9W & -0.03116 & 0.64642 & -0.11099 & 0.69198 & 0.9469 \\
S11 & -0.04926 & 0.75423 & -0.11858 & 0.78146 & 1.0861 \\
L15 & -0.04964 & 0.86742 & -0.13958 & 0.83613 & 1.2048 \\
L18W & -0.04578 & 0.87502 & -0.15706 & 0.91297 & 1.2646 \\
L24 & -0.04736 & 1.1095 & -0.099424 & 1.0903 & 1.5556 \\
\hline
\end{tabular}

Table 6 also shows the number of false detections against the number of detected sources in each band. As shown in the table, there are $45(0.5 \%)$ false detections, whereas the previous work estimated as many as $113(1.6 \%)$ false detections. For detections in two or more bands, our catalogue is expected to contain only 19 fake sources, which corresponds to $0.2 \%$. We conclude that a detection with multiple MIR bands in our catalogue is reliable at $99.8 \%$.

In the previous NIR catalogues, the false-detection rate was dominated by artificial sources, but the number of the fake sources was never estimated. We examined this using the optical catalogues (see also Sect. 4.6) under the assumption that these optical catalogues are deep enough, assuming that NIR objects with no counterparts can be regarded as fake sources. As a result, the number of unmatched objects in the previous N2, N3, and N4 band catalogues are 3564, 4338, and 4489. In contrast, as shown in Table 5, the estimated number of fake sources in our N2, N3, and N4 catalogues are only 5, 3, and 0, respectively. Therefore we conclude that the reliability of our catalogue has been much improved from the previous work.

\subsection{Astrometric accuracy}

The accuracy of the astrometric coordinates was estimated using the CFHT/MegaCam catalogue (Oi et al., in prep.), whose positional accuracy is better than 0.2 arcsec. Since the detection limit of the CFHT/MegaCam catalogue is $24 \mathrm{mag}$ in $z^{\prime}$ band, it is deep enough to cross-match with our catalogue to determine the astrometric accuracy. We cross-matched our catalogue with the MegaCam catalogue using a search radius of 5 arcsec and then calculated the differences in their positions. In doing so we did not use any objects with multiple counterparts. The results are summarised in Table 8 and Fig. 8. As shown in the figure and the table, the positional accuracy of AKARI sources is better than 2 arcsec.

\subsection{Optical identification}

To identify the optical counterparts, we created a ground-based catalogue using catalogues based on the CFHT/MegaCam $z^{\prime}$ band (Oi et al., in prep.), the WIRCam $K \mathrm{~s}$ band and the Subaru/S-cam $z^{\prime}$ band. The $K$ s band catalogue was created with the same method as the MegaCam catalogue, except that the base of the catalogue is the $K \mathrm{~s}$ band. The $\mathrm{S}$-cam catalogue covers only $40 \%$ of the NEP-deep field, while MegaCam and WIRCam cover almost the entire field. The limiting magnitude of these catalogues are $\sim 24,23$, and $26 \mathrm{AB}$ mag. To avoid any duplication we entered all MegaCam $z^{\prime}$ objects in the ground-based catalogue, then added the $K$ s objects without a 

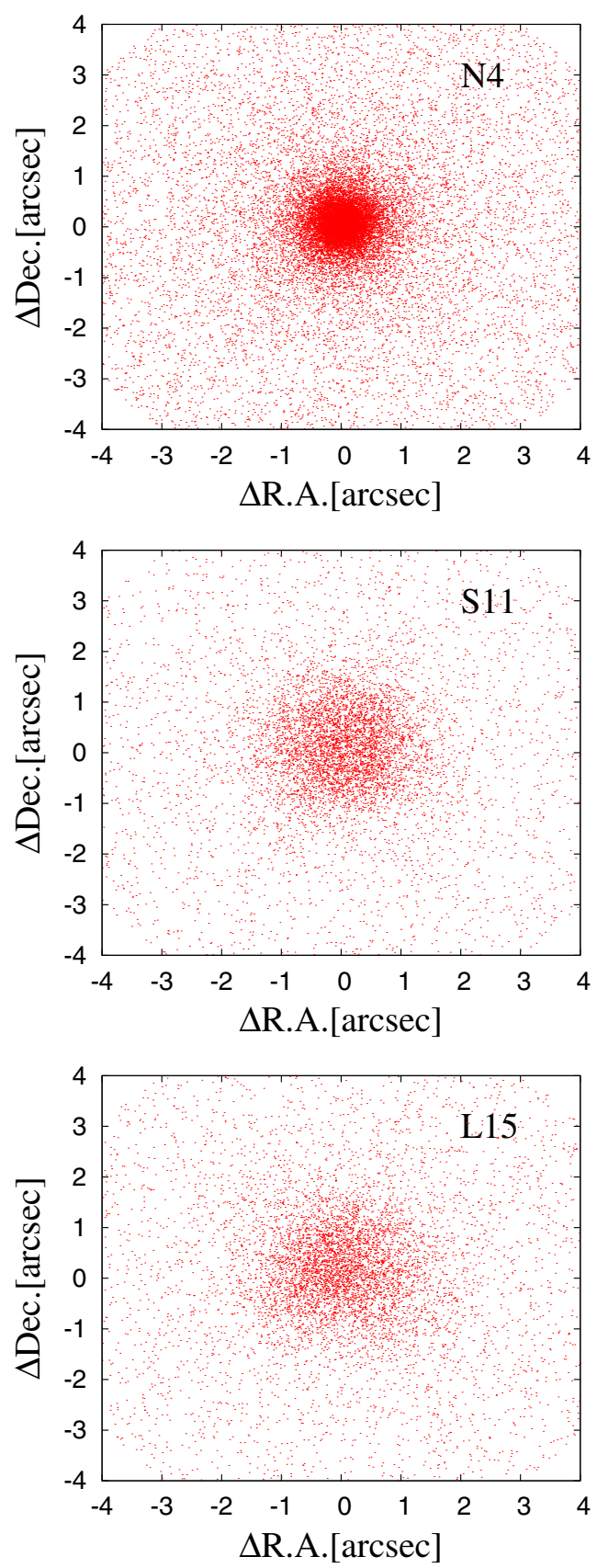

Fig. 8. Positional accuracy of the N4, S11, and L15 bands with the MegaCam $z^{\prime}$ band.

neighbour in the MegaCam $z^{\prime}$ objects within 1 arcsec. Finally, any S-cam objects without a neighbour in either the MegaCam $z^{\prime}$ or WIRCam $K$ s sources were entered. Both entries based on the MegaCam $z^{\prime}$ and WIRCam $K$ s band contain the $u^{*}, g^{\prime}, r^{\prime}, i^{\prime}, z^{\prime}$ bands from MegaCam and $Y, J, K$ s bands from WIRCam, while the catalogue based on the $S$-cam contains the $B, V, R c, i^{\prime}, z^{\prime}$, and NB711 bands. Finally, MegaCam $z^{\prime}$ or WIRCam $K$ s objects with $\mathrm{S}$-cam counterparts, found within a radius of 0.7 arcsec, also include magnitudes for the $\mathrm{S}$-cam bands.

We compared the MIR catalogue with the ground-based catalogue within a search radius of 3.0 arcsec, corresponding to $\sim 3 \sigma$ of the astrometric accuracy. According to this, 7022 objects have a single counterpart, 1572 objects have multiple counterparts, and 966 objects have no counterpart. The objects with multiple counterparts could be blended sources, and it is possible that the flux of each band is derived from different objects, because the MIR catalogue is based on the $\chi^{2}$ image, whose effective wavelength coverage is much broader. Therefore optical counterparts of these objects were searched again from the coordinates based on each band. When multiple objects were found, all of them were listed in the catalogue with a merged flag for the MIR flux. When one object was within 1 arcsec and others were separated by 2 to 3 arcsec, only the closest candidate was regarded as a counterpart. With this process, 20 MIR sources had the following problem: although the counterparts were found from coordinates from some bands, they were not found in other bands. We did not include these objects because they might be different objects. As a result, there are 11349 objects in the catalogue, 7245 of which have a single optical counterpart, 966 have no counterpart, and 3138 have a merged flag of MIR flux.

The NIR catalogues were also matched with the groundbased catalogue with a similar process. Optical objects were searched within a radius of 2.1 arcsec, corresponding to $\sim 3 \sigma$ of the astrometric accuracy. When multiple objects were found, all of them were listed in the catalogue with a merged flag for the NIR flux. When one object was within 0.7 arcsec and others were within 1.4 to 2.1 arcsec, only the nearest candidate was regarded as the counterpart. In total, 25196 objects were listed in the NIR catalogue, 3925 of which have a merged flag for the NIR flux.

Finally, the MIR and NIR catalogues were merged using the ground-based catalogue ID. Since the positional accuracy of the ground-based catalogue is much better than AKARI/NIR ones, the MIR and NIR catalogues can be merged more reliably using the ground-based catalogue ID. There are 27270 sources in the final catalogue.

\subsection{Comparison with previous catalogues}

Previous versions of the NEP-deep catalogues have been created by Wada et al. (2008) and Takagi et al. (2012). The former created nine single-band catalogues, the latter a MIR-merged catalogue with NIR photometry. A comparison between these previous catalogues and our revised catalogue is necessary to assess the merits and the demerits in our analysis. In the following we compare our NIR catalogue with that of Wada et al. (2008) and our MIR catalogue with that of Takagi et al. (2012).

The left panel of Fig. 9 shows the ratio of our flux to the previous flux against our flux for objects detected in both the previous catalogues and ours. An object in the previous catalogue whose coordinate agreed within 2 arcsec was regarded to be the same object. From top to bottom, fluxes from N3, S11, and L15 bands are compared. Our fluxes and the previous fluxes show slight differences; our N3 fluxes are $\sim 10 \%$ higher than the previous ones, while our L15 fluxes are a few percent fainter. These differences may be the consequence of the flux calibration. If this is the case, our flux measurements are more reliable because our calibration was optimised for our analysis. On the other hand, other effects may contribute. In N3 band, the method of sky subtraction can affect the flux measurement. The previous work applied a median filter with a box size of 21 pixels for the sky subtraction. This box size is not large enough and the outskirts of the PSF would be subtracted as sky background. Hence the previous work may have underestimated the source fluxes. In contrast, we applied a median filter with a box size of as large as 31 pixels; in addition, we had masked objects beforehand. Note that this effect is strongest in the NIR band because the sky background is not as high compared with the brightness of the objects. The difference in the L15 bands can 

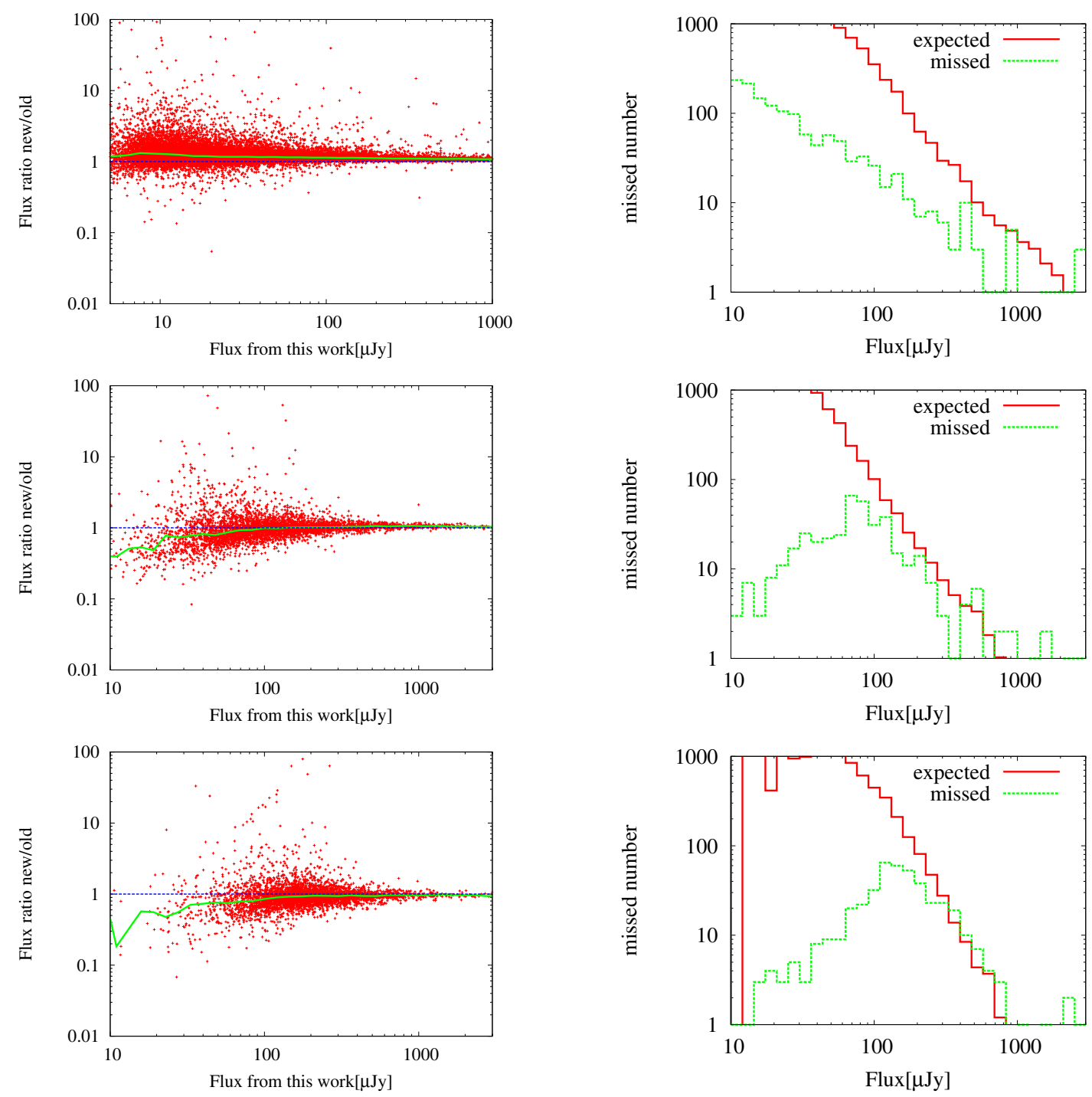

Fig. 9. Comparison between the new and old catalogues. From top to bottom, the N3, S11, and L15 catalogues are compared. Left: comparison of the fluxes for sources detected in the new and old catalogues. The green solid lines show the median line, blue dashed lines show line of unity. Right: the green dashed lines show the flux distribution of previous catalogue sources that are not detected in this work. The red solid lines show the expected number of missing objects, calculated from the completeness.
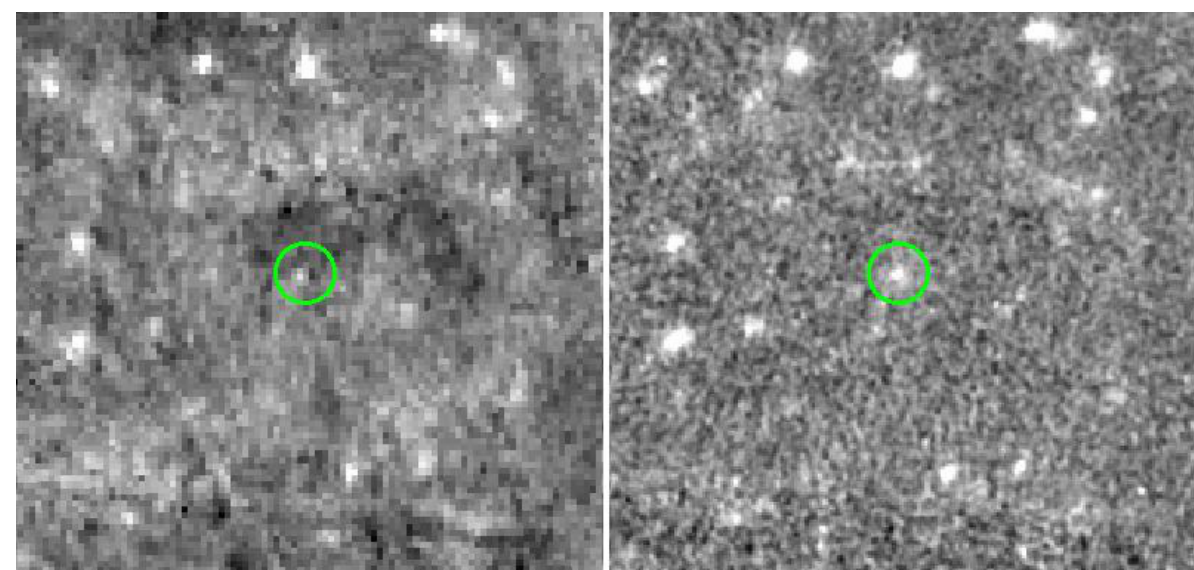

Fig. 10. Example of an object that is 60 times brighter than the previous work in the L15 band. The left panel shows the previous image, in which the object seems to be in the valley of the stray light. This could be the reason for the flux difference. be a consequence of the uncertainty in flat-fielding, because flat frames in the previous work can overcorrect the flux of a point source by $\sim 10 \%$ (Arimatsu et al. 2011).

In the comparison of the S11 and L15 bands, some of our objects are more than ten times brighter than the previous catalogue entries. We visually checked some of them and found that these objects were very faint in the previous images. The example images are shown in Fig. 10. In addition, the previously calculated WCS was not as accurate and higher pixel values were clipped when the images were combined, or the sources are in 
the stray-light valley (see also Sect. 3.3). In this work, we registered the WCS information very carefully, and the stray light was subtracted. Hence, our values are more reliable for these objects than previous versions of the catalogue.

In the right panel of Fig. 9 the green dashed lines show the flux distribution of the previous catalogue version sources that are not detected in ours. The red solid lines show the expected number of missed objects, calculated from the completeness of each band. Since our detection threshold of the NIR sources is much stricter than any previous work, many objects are missed in our catalogue. Conversely, the previous catalogues have many fake sources instead. As we described in Sect. 4.1, for the MIR catalogue only objects detected at the same position within 2.5 arcsec from the reference sample are regarded as real sources. Although this criterion was set to avoid a false extraction due to a chance coincidence, some objects were missed in our catalogue. As these were explained by the incompleteness, it does not significantly affect statistical completeness analysis.

\subsection{Catalogue description}

We have to note again that objects with multiple counterparts from the ground-based catalogue are duplicated because all of the counterparts are listed with a merged flag of AKARI flux. In this case coordinates are based only on ground-based catalogue. The catalogue entries are

(1) the source ID;

(2) the source name, which is based on the coordinates;

(3) and (4) RA and Dec.

One can know the derivation of the coordinates by the groundbased catalogue band in the next column. If no ground-based catalogue band exists, the coordinates are based on AKARI $/ \chi^{2}$.

(5) Ground-based catalogue band. It indicates which band is used as the base band; $\mathrm{Z}, \mathrm{K}$, and $\mathrm{S}$ indicate MegaCam $z^{\prime}$, WIRCam $K$ s and S-cam $z^{\prime}$ band catalogues, respectively.

(6) Ground-based ID. It is only available for MegaCam $z^{\prime}$ band. (7) AKARI $/ \chi^{2}$ ID. Some objects have the same $\chi^{2}$ ID, which indicates that they have multiple optical counterparts.

(8) and (9) RA and Dec from AKARI/ $\chi^{2}$.

This exists when MIR sources have only one or no ground-based counterpart. No coordinates based on the NIR bands are available because NIR objects always have counterparts from groundbased catalogue, and listing all the coordinates based on N2, N3, and $\mathrm{N} 4$ bands is complicated.

(10)-(24) MegaCam $u^{*}, g^{\prime}, r^{\prime}, i^{\prime}, z^{\prime}$ magnitudes, errors and flags. The flag is the same as in Oi et al.; 0: general. 1: it is blended, but might be deblended in an other band. 2: it could be blended. (25)-(33) WIRCam $Y, J, K$ s magnitudes and errors. The flag is the same as for MegaCam.

(34)-(51) S-cam $B, V, R s, i^{\prime}, z^{\prime}$, NB711 band magnitudes and errors. The flag is the same as for MegaCam.

(52)-(78) AKARI N2, N3, N4, S7, S9W, S11, L15, L18W, L24 flux density and errors in terms of Jy. The flag is the same as for MegaCam.

(79) Stellarity from the MegaCam catalogue. Objects with no counterparts from MegaCam have no value, even if they have other counterparts from WIRCam or S-cam.

(80) Angular separation from optical counterparts to $\chi^{2}$ objects. This exists for MIR sources that have only one optical counterpart.

All magnitudes are provided in the AB system. Nondetection is indicated by flux or magnitude values of -1 or -99 .

\section{Summary}

We presented a revised near- to mid-infrared catalogue for the AKARI NEP-deep survey. All images were re-analysed with new image analysis methods to remove the scattered light in the detectors and camera optics, the stray-light from the Earth limb, and the artefact patterns in the images by creating templates for their patterns. Artificial sources caused by bright objects were removed using their characteristics or were otherwise visually masked. In addition, flat frames were revised to improve the accuracy of the flux measurement by as much as $\sim 10 \%$. Moreover, we added other ancillary AKARI images in the NEP region, increasing the number of images by $\sim 15 \%$. For the MIR source extraction, we created detection images using all six bands with a $\chi^{2}$ method, in which the sky noise was significantly reduced. For the NIR catalogue, we considered only objects with optical counterparts to reduce the number of false detections. For our optical catalogues, we used catalogues based on the CFHT/MegaCam $z^{\prime}$ band, WIRCam Ks band and Subaru/S-cam $z^{\prime}$ band.

We evaluated the detection limit, the number of detected sources, and the reliability of the resulting catalogue. It was concluded that the detection limit of the MIR catalogue has been improved by $\sim 20 \%$, and while the addition of the images can contribute at most $\sim 7 \%$; this indicates that our image analysis and the source extraction method contributes in a major way to the improvement of the catalogue, although we were unable to determine which method dominates the improvement. As a result, the $5 \sigma$ detection limits in our catalogue are $11,9,10,30,34,57,87,93$, and $256 \mu \mathrm{Jy}$ in the N2, N3, N4, S7, S9W, S11, L15, L18W, and L24 bands, respectively. The corresponding $50 \%$ completeness limits for these bands are $56,55,38,29,34,50,86,94$, and $247 \mu \mathrm{Jy}$, respectively. The number of MIR objects was increased by 2000 to 9560 compared with Takagi et al. (2012). Furthermore, the false-detection rate has been much reduced, making the new catalogue reliable at $99.7 \%$ even in the single-band detection.

Optical counterparts were identified from the optical catalogue. Objects with multiple counterparts are listed in the catalogue with an upper limit for the AKARI flux. In total, 27770 objects were listed in our catalogue, 11349 of which have MIR fluxes.

The comparison with the previous and the revised catalogues helped us to understand the merits and the demerits of our analysis. Our fluxes and those of the previous catalogue differ slightly by up to $10 \%$, which might be caused by the difference in flux calibration. Some objects seemed to recover more fluxes, since we registered the WCS information very carefully, or the stray light from the Earth limb was subtracted. In addition, the comparison implied that the flat-fielding accuracy was improved. Some objects were not detected in our catalogue, even though they were listed in the previous catalogue, which might be explained by incompleteness in our detection, and hence does not significantly affect our results.

The new catalogue is much improved in the detection limit, the number of sources and the reliability of the source extraction, and will provide an extremely valuable database for studying the activity in galaxies up to $z \sim 2$.

Acknowledgements. We would like to thank the AKARI IRC team members for their support in this work. We also thank the referee who gave us useful comments. The AKARI NEP-deep survey project activities are supported by JSPS grant 23244040 . 
A\&A 559, A132 (2013)

\section{References}

Arimatsu, K., Onaka, T., Sakon, I., et al. 2011, PASP, 123, 981 Beers, T. C., Flynn, K., \& Gebhardt, K. 1990, AJ, 100, 32

Bertin, E., \& Arnouts, S. 1996, A\&AS, 117, 393

Cohen, M. 2003, in The Calibration Legacy of the ISO Mission, eds. L. Metcalfe,

A. Salama, S. B. Peschke, \& M. F. Kessler, ESA SP, 481, 135

Cohen, M., Witteborn, F. C., Carbon, D. F., et al. 1996, AJ, 112, 2274

Cohen, M., Walker, R. G., Carter, B., et al. 1999, AJ, 117, 1864

Cohen, M., Megeath, S. T., Hammersley, P. L., Martín-Luis, F., \& Stauffer, J. 2003a, AJ, 125, 2645

Cohen, M., Wheaton, W. A., \& Megeath, S. T. 2003b, AJ, 126, 1090

Fazio, G. G., Hora, J. L., Allen, L. E., et al. 2004, ApJS, 154, 10

Goto, T., Takagi, T., Matsuhara, H., et al. 2010, A\&A, 514, A6

Jarrett, T. H., Cohen, M., Masci, F., et al. 2011, ApJ, 735, 112

Kawada, M., Baba, H., Barthel, P. D., et al. 2007, PASJ, 59, 389

Le Floc'h, E., Papovich, C., Dole, H., et al. 2005, ApJ, 632, 169

Lorente, R., Onaka, T., Ita, Y., et al. 2008, AKARI IRC Data User Manual v.1.4, http://www.ir.isas.jaxa.jp/ASTRO-F/Observation
Masci, F. J., \& Fowler, J. W. 2009, in Astronomical Data Analysis Software and Systems XVIII, eds. D. A. Bohlender, D. Durand, \& P. Dowler, ASP Conf. Ser., 411, 67

Matsuhara, H., Wada, T., Matsuura, S., et al. 2006, PASJ, 58, 673

Murakami, H., Baba, H., Barthel, P., et al. 2007, PASJ, 59, 369

Onaka, T., Matsuhara, H., Wada, T., et al. 2007, PASJ, 59, 401

Pérez-González, P. G., Rieke, G. H., Egami, E., et al. 2005, ApJ, 630, 82

Rieke, G. H., Young, E. T., Engelbracht, C. W., et al. 2004, ApJS, 154, 25

Sakon, I., Onaka, T., Wada, T., et al. 2007, PASJ, 59, 483

Skrutskie, M. F., Cutri, R. M., Stiening, R., et al. 2006, AJ, 131, 1163

Szalay, A. S., Connolly, A. J., \& Szokoly, G. P. 1999, AJ, 117, 68

Takagi, T., Ohyama, Y., Goto, T., et al. 2010, A\&A, 514, A5

Takagi, T., Matsuhara, H., Goto, T., et al. 2012, A\&A, 537, A24

Tanabé, T., Sakon, I., Cohen, M., et al. 2008, PASJ, 60, 375

Wada, T., Matsuhara, H., Oyabu, S., et al. 2008, PASJ, 60, 517

Werner, M. W., Roellig, T. L., Low, F. J., et al. 2004, ApJS, 154, 1

Wright, E. L., Eisenhardt, P. R. M., Mainzer, A. K., et al. 2010, AJ, 140, 1868 